\title{
Gender x Culture: A pilot project exploring the study abroad experiences of trans and gender expansive students
}

\author{
Taylor Michl \\ Kelsey Pegg \\ Amanda Kracen \\ Webster University
}

\begin{abstract}
:
As gender is socially constructed, gender attitudes, expressions, expectations, and identities may differ based on their sociocultural contexts. This project sought to understand interactions between gender and culture through the experiences of three undergraduate study abroad students who are trans and/or gender expansive. Utilizing the thematic analysis method, data from a focus group and individual follow-up interviews were analyzed; seven themes emerged from the data collected. As study abroad has become an increasingly normalized aspect of the American undergraduate experience, institutions must consider the ways study abroad may uniquely affect certain student populations. This project explored some of the unique joys (community building, self-exploration, cultural insights) and hardships (gender-policing, isolation, sexual violence) of study abroad for trans and gender expansive students, identified areas for further research, and suggested specific methods through which institutions can provide support to these students.
\end{abstract}

\section{Relevant Gender and Sexuality Terminology}

Although not an exhaustive list, the following are definitions of the genders and sexual identifications referenced in this paper. Individuals may (but do not necessarily) identify simultaneously with more than one of the identities listed. Gender expansive individuals identify and/or express their genders in ways that broaden cultural understandings of gender. This identity can encompass people who are genderqueer, nonbinary, gender nonconforming, and otherwise not cisgender. Genderqueer people sometimes (but do not always) identify and/or express their genders outside of the gender binary. They may also (but do not necessarily) identify and/or express their genders as fluid. Nonbinary individuals identify and/or express their genders in ways that fall outside of the gender binary. Nonbinary can be (but is not always) used as an umbrella term. Gender nonconforming people identify and/or express their genders in ways that do not align with societal conceptions of gender. Transgender (or trans) individuals are people whose genders differ from the sex they were assigned at birth. This can include people whose genders are opposite the sex that they were assigned at birth and those whose genders fall outside of, in between, or otherwise differ from the normative gender binary as defined by society. Cisgender individuals are people whose genders align with the sex they were assigned at birth. Gender-policing is the act of forcing normative ideas of gendered behavior and expression on individuals, especially those who violate gender norms. Gender-policing may occur through verbal comments, nonverbal behaviors, or physical 
violence. Queer is a historically derogatory term that has been re-appropriated by many LGBTQ+ (lesbian, gay, bisexual, transgender, queer/questioning, and more) individuals who now utilize it as an identity label. This term may refer to gender and/or sexuality and can function as an umbrella term for people who are not heterosexual and/or not cisgender. Sapiosexual is a sexual identity term used by individuals whose experience of sexual and/or romantic arousal is stimulated by the intelligence of their partner(s). Pansexual is a sexual identity term used by individuals who experience sexual and/or romantic attraction to people of all genders.

\section{Introduction}

\section{Literature Review}

With adequate communities, support, and resources, study abroad can be an intensely enriching experience for trans and gender expansive (TGE) students. In fact, Bryant and Soria (2015) found that many LGBTQ+ students are motivated to study abroad, in part, to explore their gender and sexual identities. Along with this increased self-understanding, a 2012 study found that study abroad students returned to their home countries with perceived improvements in the areas of patience, flexibility, and cultural sensitivity, as well as the increased ability to "navigate daily routines in a new environment" and make complex language and cultural comparisons (Cisneros-Donahue et al., 2012, p. 72). In addition, research conducted by the Institute for International Education (2017) found that more than half of students felt that their study abroad experiences somehow contributed to job offers they received post-graduation. The same study indicated that studying abroad opened new career opportunities for study abroad alumna of which they were previously unaware or had not considered.

However, along with lesbian, gay, and bisexual people (LGB), members of trans and gender expansive (TGE) communities may experience unique stressors that affect their physical and mental health (Meyer, 2015). According to Meyer (2003), the concept of "minority stress" points to the overabundance of stress which individuals who inhabit minority or marginalized social positions experience as a direct consequence of their stigmatized identities. Meyer identified two interconnected types of stressors within the minority stress model: distal and proximal stressors (2003). Distal (external) stressors may be experienced by TGE individuals in the forms of rejection, discrimination, harassment, and violence within various contexts (i.e., work, school, medical, governmental, and family settings) (Matsuno \& Israel, 2018). As a result of distal stressors, TGE people may encounter proximal (internal) stressors such as fear of potential and/or continued discrimination or violence and "transnegativity" or, according to Matsuno and Israel (2018), "holding negative beliefs about their identity and about others who share this identity as a result of social stigma placed on transgender identities" (636). Although the minority stress model has been adapted and applied to TGE communities (Meyer, 2015; Matsuno \& Israel, 2018), it previously pertained to LGB communities exclusively (Meyer, 1995, 2003).

In their study about the Gender Minority Stress and Resilience (GMSR) Measure, Testa, Habarth, Peta, Balsam and Bockting (2015) noted that TGE individuals often experience stressors that are distinct from those experienced by cisgender LGB people. Gender is often assumed immediately through physical observations and, in many cultures, gender assumptions dictate the ways in which people interact. Therefore, cisgender LGB people sometimes occupy positions of relative privilege in comparison to those who are TGE in their abilities to exercise control over to 
whom and how they 'come out.' In other words, “in some ways there is no option for 'don't ask, don't tell' regarding gender” (Testa et al., 2015, p. 67).

During an undergraduate study abroad experience, students are provided with the opportunity to interact with individuals whose cultural identities differ from their own. While this may be an enriching experience for some, it may also be interpreted as a minority stressor for TGE students. It is possible that, during these interactions, TGE students may be concerned about how they are perceived by new people and possible violence they may experience, compounded by the general stress of navigating unfamiliar locations and cultures. According to the United States Transgender Survey (2015), 46\% of transgender individuals were verbally harassed and $9 \%$ experienced physical attacks in the United States as a result of their gender; these figures increase dramatically for transgender and gender expansive people of color in the U.S. It is logical, then, that TGE students may feel even more vulnerable to violence and harassment when entering unfamiliar cultures, especially if they are not equipped with resources regarding LGBTQ+ history, laws, and social stigma in their host countries. In addition, if TGE study abroad students do not have knowledge of or access to LGBTQ+ communities in their host countries, they could feel alienated.

For TGE individuals who "[occupy] positions of token or solo status," which may be the case if they are the only trans or gender expansive study abroad student from their institution, minority stressors may further increase as this often makes them more vulnerable to decreased self-esteem resulting from self-comparison to the dominant group (Crocker \& Major, 1989, p. 621). When the sense of belonging and self-esteem decreases and that of victimization and stress increases, TGE individuals may think that their trans and gender non-normative identities present "a burden to society" (Hendricks \& Testa, 2012, p. 464). However, according to Crocker and Major (1989), "when the stigmatized are in solo status situations, even a small cohort of similarly stigmatized peers may be sufficient to enable self-protective strategies to function effectively" (621). Moreover, when TGE study abroad students have access to communities of others who share similar stressors, they may be better equipped to handle gender-based minority stress.

As research has shown overwhelming benefits to study abroad for all undergraduate student populations, it is imperative that institutions support the safety and wellbeing of trans and gender expansive study abroad students. According to the Association of International Educators, more than 325,000 students enrolled in U.S. higher education institutions studied abroad during the 20152016 academic year and about 10\% of all United States college graduates have participated in a study abroad program (Farmer, 2016). As study abroad has become an increasingly normalized aspect of the United States undergraduate experience, far more research is needed to understand the joys, hardships, and needs of TGE students who participate in international study. Marijuan and Sanz (2018) also call for more extensive research on LGBTQ+ study abroad experiences, as living internationally can be extremely gendered in nature.

\section{The Current Study}

Building on the need for investigation of the relationships between gender and culture as they relate to study abroad, this qualitative research project sought to understand the experiences of undergraduate students who have studied abroad and are transgender and/or gender expansive. As cultural values affect the treatment of gender non-normative communities in different countries, this 
research both highlights queer students' experiences and yields insights about cultural differences. This research, rather than focusing specifically on one location, sought to understand the study abroad experiences of trans and gender expansive students more generally. As this population is especially vulnerable to verbal, physical, and sexual assault in the US, stepping into new cultures that may or may not be accepting of their identities can be anxiety-provoking. The U.S. Transgender Survey (2015) found that $24 \%$ of transgender people who were 'out' and/or perceived by others as trans were verbally, sexually, or physically harassed in college and vocational school settings. With this in mind, it is reasonable to assume that TGE students may be at heightened risk for violence and harassment while studying abroad, and this worry is exacerbated by their potential lack of familiarity with their international location. A literature review revealed scarce research focused specifically on TGE students who study abroad. Therefore, this pilot study, intended to be explorative in nature, contributes to the scare research literature and identifies issues for further research. It is the researchers' hope that this study will provide TGE students with some issues to consider when contemplating study abroad, as well as a shared narrative on this complex topic. In addition, findings allow colleges and universities to better understand the ways in which gender and culture have intersected to affect some students' educational experiences abroad and, therefore, identify the necessary support and resources to provide to students.

\section{Ethics}

The Institutional Review Board at Webster University granted approval for the current study. Participants provided written informed consent prior to the focus group and follow-up interviews. Funding for this project was provided by Webster University's Student/Faculty Collaborative Research Grant; participants were compensated with a $\$ 25$ gift of their choice.

The research team members recognize that LGBTQ+ and, more specifically, trans and gender expansive (TGE) communities have historically been subject to exploitation in medical, psychological, and other research areas. For this reason, the research team consulted resources and engaged in dialogue surrounding the specific ethical considerations of conducting research with TGE populations. The procedures of the current study were conducted with this history in mind, and researchers made every effort to reduce the potential for participant exploitation. Issues such as participant internet accessibility, compensation, and confidentiality; focus group and interview environments and questions; and researcher biases (Meezan \& Martin, 2009) were considered throughout participant recruitment, data collection, and data analysis. The researchers do not seek to present trans and gender expansive study abroad students as a monolithic population in this study. Rather, the aim was to provide a shared narrative from a small group of three individuals on this complex topic for past, present, and future TGE study abroad students.

\section{Method}

\section{Study Design}

This was a qualitative study using a focus group design and data were analyzed using the thematic analysis approach (Braun \& Clarke, 2006; Clarke \& Braun, 2013). Thematic analysis (Braun \& Clarke, 2006; Clarke \& Braun, 2013) facilitates the identification of themes in a dataset and does not require a specific theoretical or disciplinary framework. Braun and Clarke (2006, 2013) posit that theoretical flexibility is a notable advantage of thematic analysis and have created guidelines for utilizing the method that maintain this flexibility while also standardizing its process in order to 
ensure accuracy and reliability. As the research team for this study consisted of three individuals of varying academic and social backgrounds, thematic analysis allowed team members to collaboratively utilize their individual expertise in order to engage in a thorough investigation and yield rich results.

\section{Sample Selection and Characteristics}

\section{Participants.}

Participants were recruited through purposeful and snowball sampling in a Midwestern city. The study announcement was emailed to 1) professors of sociology, anthropology, psychology, women \& gender studies, and other related fields at local undergraduate institutions, 2) study abroad directors and program staff at local undergraduate institutions, 3) relevant local LGBTQ+ community organizations, and 4) potentially interested personal contacts of the researchers. Email recipients were asked to forward the study information to others who could be interested. Interested participants were emailed the informed consent and demographics forms and asked to complete an online questionnaire to indicate their availability for the focus group. The purpose of the current study was explained as an exploration of "the experiences of undergraduate students who have studied abroad and are transgender and/or gender expansive (which includes people who are genderqueer, nonbinary, gender nonconforming or otherwise not cisgender).”

In order to be eligible for participation in the current study, participants had to (a) be over the age of 18 years, (b) have studied abroad outside of the United States, (c) have been enrolled in an undergraduate program within the last three years, and (d) be transgender and/or gender expansive. Individuals who met these requirements and were interested in participating were instructed to contact the research team in order to receive the informed consent and demographics forms, ask questions about the study, and schedule the focus group. Seven individuals expressed interest in participating; four were unable to participate due to eligibility requirements and scheduling conflicts. Three eligible participants provided consent and participated together in one focus group; subsequently each person completed a follow-up phone interview with the first author. Participant characteristics are displayed in Table 1. Participants $(\mathrm{N}=3)$ ranged in age from 21-23. All participants studied abroad one time and the duration of their stay abroad ranged from five to 12 months. Two participants studied in Europe and one studied in Asia. Of note, the first author knew two participants personally, while the other two research team members had no personal connections to the participants.

Table 1. Participant characteristics. ${ }^{1}$

\begin{tabular}{|l|l|}
\hline Racial Identities & Latinx/Hispanic; Caucasian/white \\
\hline Genders & Non-binary; female $^{2}$ \\
\hline Pronouns & They/él; she/her ${ }^{2}$ they/them \\
\hline
\end{tabular}

\footnotetext{
${ }^{1}$ To protect the anonymity of participants, demographic information is presented altogether rather than individually.

${ }^{2}$ One participant, who used she/her pronouns and identified their gender as "female" on the demographic form and during all steps of data collection, now identifies as non-binary and uses they/them pronouns. In order to embrace the fluidity of gender and to respect participants' ongoing journeys, they/them pronouns are used throughout the article in reference to this participant. However, as this individual's responses and reflections may have been affected by their pronouns and gender at the time of the study, the authors have included their previous gender and pronouns in this table. The authors acknowledge that identity is ever-evolving, and all participants' genders,
} 


\begin{tabular}{|l|l|}
\hline Sexual Identities & Sapiosexual/pansexual; queer \\
\hline Age (Range) & $21-23$ \\
\hline Study Abroad Locations & Europe; Asia \\
\hline Study Abroad Living Arrangements & With host family; alone; with other students \\
\hline Study Abroad Duration (Range) & Five to 12 months \\
\hline
\end{tabular}

Research team.

The research team was comprised of three individuals, ranging in age from 22 to 43 . All researchers self-identify as non-Hispanic Caucasian. The first author is a recent graduate with a Bachelor's degree in Women, Gender, and Sexuality Studies and Spanish, although this study was completed during their undergraduate career. Another research team member has a Master's degree in Clinical Mental Health. Finally, another team member is an associate professor in an undergraduate psychology department. One of the researchers is a gender expansive person, one is a nonbinary trans person, and one is a cisgender woman. It is significant that two of the research team members on the current study, including the first author, are trans and/or gender expansive. The research team engaged in discussions about their own identities, perspectives, and biases before data collection and during data analysis, deciding that it was appropriate that the two researchers who identify as transgender and/or gender expansive should be present for the focus group and followup interviews (one facilitated the focus group and follow-up interviews, one observed the focus group). This decision was made to increase the comfort levels of participants in sharing their experiences navigating study abroad as gender non-normative people. In addition, the investigators' identities have aided in the collection, analysis, and dissemination of data surrounding complex identities and experiences.

\section{Data Collection}

Demographic form.

Each participant was asked to fill out a short demographic form before the focus group. Participants provided their contact information, age, gender, pronouns, sexuality/sexual identification, race, and college or university that they attend(ed). In addition, they were asked about their relationship status, parental status, highest level of education, study abroad duration and location, and living situation while abroad.

Focus group discussion.

The focus group was facilitated by the first author and observed by the second author, both of whom are transgender and/or gender expansive. Participants were provided with the list of focus group questions prior to the focus group to familiarize themselves with the discussion topics; the focus group questions are listed in the Appendix. As the research team aimed to increase the comfort levels of participants, the facilitator asked that participants approach the focus group as a conversation between the participants and facilitator. Participants were encouraged to ask questions and make comments in response to others' experiences. Light refreshments were provided. With permission, the focus group was audio recorded. Prior to the focus group, participants were read a consent reminder and asked for verbal consent. The focus group lasted 120 minutes. The second author took notes regarding the group process.

pronouns, and conceptions of their own experiences may have shifted since the publication of this article. 
Follow-up interviews.

After the focus group, each participant scheduled an individual follow-up phone interview, which took place one to two weeks later. The purpose of the call was for participants to amend or clarify any of the comments they made during the focus group, add other relevant information, and reflect on their focus group experience. They were asked two questions: (1) Is there anything that was said during the focus group that you would like to elaborate on or clarify? and (2) Is there anything that was not covered during the focus group regarding your study abroad experience and/or your gender that you would like to discuss? Participants were read a consent reminder and asked for verbal consent; phone calls were audio recorded. The follow-up interviews ranged from four to ten minutes in length.

Transcription.

The first author transcribed the focus group and follow-up interviews, and transcripts were sent to the participants to review and amend any responses as they deemed necessary. The research team provided this opportunity for revision in order to ensure that participants, especially as members of marginalized populations, were able to exercise control and autonomy over their own narratives. One participant suggested minor revisions for the purpose of clarification.

\section{Analysis Strategies}

Coding.

Data were analyzed using the Thematic Analysis method (Braun \& Clarke, 2006; Clarke \& Braun, 2013), in which information is separated into broad themes. All research team members participated in each step of analysis and held meetings to discuss the process and reach consensus.

According to Braun and Clarke (2006, 2013), Thematic Analysis requires the completion of six steps. The first step seeks to familiarize investigators with the data and identify their initial impressions. The second step is the creation of codes (short summarizing phrases) for all data. This step allows investigators to condense the focus group and interview content to its core concepts. In our study, the first author created codes for the focus group transcript and the other researchers reviewed and amended the codes. Later, each researcher was assigned one follow-up interview transcript for which they created codes. The team collaboratively reviewed and amended the codes yielded from the follow-up calls. Altogether, 171 codes were identified within the focus group and follow-up interview transcripts.

Codes were then divided into themes and subthemes, which is the third step of Thematic Analysis. The research team identified themes by printing and cutting out each code separately and spreading them out on a table. Codes were grouped together based on commonalities and themes emerged. This physical grouping of codes protected against researcher bias, as researchers were not able to associate codes with participants or interview questions. Seven major themes were identified, which are displayed in Table 2.

The fourth step of Thematic Analysis assesses the themes created to ensure that they are logical, supported by the data, appropriately sized, distinct and encompassing. During this step, the research team decided to add theme F, Lacking Preparation and Institutional Support, as some data that had previously been placed in separate themes included similar concepts. After this assessment, 
the researchers collaborated to create names for and descriptions of each theme and discussed the significant details of the study to include in the written report, which represent steps five and six of Thematic Analysis.

Table 2. Themes.

\begin{tabular}{|c|c|c|}
\hline Theme & Subthemes & Topics $^{3}$ \\
\hline $\begin{array}{l}\text { 1. Navigating the } \\
\text { Gender and } \\
\text { Identity Journey }\end{array}$ & $\begin{array}{l}\text { a) The Process of 'Coming Out' } \\
\text { b) The Significance of Pronouns }\end{array}$ & $\begin{array}{l}\text { Evolving understanding of own identity; level of comfort with } \\
\text { identity; when/to whom/how to 'come out' and/or share pronouns; } \\
\text { gender-neutral pronouns in languages other than English; sacredness } \\
\text { of pronouns; establishing identity on campus; utilizing gender } \\
\text { expression to understand identity; detachment from/attachment to } \\
\text { gender }\end{array}$ \\
\hline $\begin{array}{l}\text { 2. Constructing } \\
\text { Community }\end{array}$ & None & $\begin{array}{l}\text { Relationships with other queer people in the US and lack thereof } \\
\text { abroad; relationships with genderfluid 'role models' abroad; lack of } \\
\text { information about queer communities abroad; supportive friendships; } \\
\text { effects of studying abroad on romantic/sexual relationships; formation } \\
\text { of communities abroad }\end{array}$ \\
\hline $\begin{array}{l}\text { 3. Experiencing } \\
\text { Threat and } \\
\text { Violence }\end{array}$ & None & $\begin{array}{l}\text { Instances of sexual harassment, assault, and rape abroad; disregard } \\
\text { and shaming based on race and gender from community and } \\
\text { institutions after experiences of violence; safety while traveling; more } \\
\text { violent gender-policing abroad than in US; microaggressions } \\
\text { experienced by TGE people at the hands of cisgender and TGE people } \\
\text { in US and abroad }\end{array}$ \\
\hline $\begin{array}{l}\text { 4. Managing } \\
\text { Others' } \\
\text { Assumptions } \\
\text { About Identity }\end{array}$ & None & $\begin{array}{l}\text { Questioning how racial, gender, and sexual identities are viewed by } \\
\text { others in home, school, street, and other environments abroad; } \\
\text { reacting to others' assumptions about identities; constant 'coming out' } \\
\text { in reaction to incorrect assumptions; associations between sexual and } \\
\text { gender identities made by others; navigating when to expend energy } \\
\text { on correcting others' assumptions }\end{array}$ \\
\hline $\begin{array}{l}\text { 5. Performing and } \\
\text { Policing Gender }\end{array}$ & None & $\begin{array}{l}\text { Experiences of queer men gender-policing abroad; expressing gender } \\
\text { differently based on perceived safety and risk; different gender } \\
\text { expression expectations abroad; gender-policing from romantic/sexual } \\
\text { partners; transmasculine people more able to blend in than } \\
\text { transfeminine people abroad; privilege of 'passing' as cisgender; } \\
\text { gender-policing more overt and violent abroad; expressing identity } \\
\text { through clothing choices }\end{array}$ \\
\hline $\begin{array}{l}\text { 6. Lacking } \\
\text { Preparation and } \\
\text { Institutional } \\
\text { Support }\end{array}$ & None & $\begin{array}{l}\text { Gender-segregated sleeping arrangements on school-affiliated trips; } \\
\text { support of U.S. institution in providing safe housing; conducting } \\
\text { research and asking institution for resources before studying abroad; } \\
\text { participants not given LGBTQ+-specific resources by institutions; } \\
\text { teachers abroad respecting pronouns; trans people generally othered in } \\
\text { school and work settings; minimal preparation for study abroad }\end{array}$ \\
\hline $\begin{array}{l}\text { 7. Celebrating } \\
\text { their Experiences } \\
\text { of Studying } \\
\text { Abroad }\end{array}$ & None & $\begin{array}{l}\text { Participants would study abroad again; wants to go somewhere that } \\
\text { feels less culturally 'safe' if given the opportunity to study abroad } \\
\text { again; positive, special, "magical" experiences while abroad; } \\
\text { resilience }\end{array}$ \\
\hline
\end{tabular}

${ }^{3}$ Themes were generated from the original list of codes created by the research team. Due to the large quantity of codes, they are not included here. Instead, the general topics which fall into each theme are explained. 


\section{Findings}

Seven themes were identified, as presented in Table 2.

\section{Theme 1: Navigating the Gender and Identity Journey}

Communities with marginalized identities (TGE study abroad students, for example) are often forced to engage in constant reflection regarding the ways these identities affect, alter, and relate to their experiences and interactions. Contrastingly, communities with socially privileged identities (cisgender study abroad students, for example), because they are normalized in society, often do not have to partake in this kind of emotional and cognitive labor. Although some cisgender study abroad students may be able to conceptualize their international experiences without considering their genders, TGE students may not have this privilege. Therefore, participants in this study were actively engaged in reflecting on their identities in the context of unfamiliar cultures while abroad. This theme encompasses the ways in which participants' study abroad experiences were affected by their decisions to 'come out' and/or to share pronouns as well as their evolving understandings of their own identities.

\section{Subthemes:}

\section{a) The Process of 'Coming Out'}

This subtheme included participants' decisions regarding to whom, how, and when they 'came out' during their study abroad experiences; some were skeptical of the concept of 'coming out' itself because of their already visible TGE identities.

\section{b) The Significance of Pronouns}

This subtheme is comprised of participants' decisions regarding sharing their pronouns abroad, the evolution of their pronoun choices, and their navigation of utilizing pronouns in an international cultural context.

As the duration of participants' study abroad programs was merely five to 12 months, these experiences required the accelerated development of social connections. Participants emphasized that the time constraints while studying abroad created pressure to 'come out' sooner in order to develop relationships. The ways in which participants navigated 'coming out' were diverse given that, for some, they did so daily through sharing pronouns while others described a more intentional decision-making process in regard to how and to whom they 'came out' about their gender and/or sexuality. One person described the ways their friends supported their identity in their absence:

To my understanding, my friends that semester helped me out by letting others know what my pronouns were. This eased the burden of having to always explain and introduce myself . .. I think it's extremely valuable to have even at least one person around who can vouch for you/back you up...especially in a world that's constantly trying to invalidate trans identities.

Participants also mentioned the evolution of their identities that took place during and after study abroad. One individual, who first explored their non-binary identity while they were studying abroad, used gender-neutral pronouns in English. However, the main language spoken in their host country does not have a gender-neutral pronoun alternative. They described coming to terms with this reality: 
... for me [it] was pivotal in my own journey that, hey, there are just some languages where gender-neutral pronouns doesn't work and, cool, cute, that's okay, it doesn't have to work in every language.

Another participant described a shift in their gender expression while they were studying abroad, which affected the ways they saw their own identity.

And it's interesting to see ... even the clothing choices that I wore before going [abroad], I felt like I kind of fit into, conforming ... to just a norm of what it means to be . . f feminine. So I think just evolving throughout that experience, I've kind of used style to make sense of my identity in a lot of ways . . . and I kind of like, now, being able to play around with it and kind of seeing what's comfortable, what feels empowering, what feels good.

\section{Theme 2: Constructing Community}

Participants discussed the formation and effects of their communities, as well as their social flexibility and openness (or lack thereof) during their study abroad trips. Two participants gave examples of individuals they met abroad who expressed their genders in non-normative ways and, in turn, allowed participants to build strong bonds and express their own identities in new ways.

... it really helped that there was this professor there who ... he's just a very queer man of color, very unapologetic about how he performs his gender, painting his nails, wearing clothes that weren't necessarily gender restrictive ... I learned a lot from him, honestly, like how I perform my gender now very much, I feel like is a consequence of having witnessed him perform his gender and the level of comfort that he had ... it was liberating.

Each participant communicated that their support systems in the US are largely composed of queer people, while this was not the case during their time abroad. One participant suggested that queer students should find qualities that connect them to others abroad outside of queer identities, as it may be more difficult to locate queer communities abroad. Another participant noted that their queer identity added a layer of fear to branching out of their existing social group:

I knew that [my friends abroad] just, like, 'got' [my identity] to a certain degree that made me feel safe, and I guess going out of that kind of felt scary to a certain degree because I was, just besides gender, in a whole other culture and country.

Participants considered many of their relationships abroad to be special and fulfilling bonds, and expressed the importance of both queer and chosen family:

[Cooking together] was very sweet and simple, and [my friends and I] would cook a big meal together ... Even when I lived in the dorms . . . we never ate alone . . . so we would cook together and we'd have other people from the dorms come down and I would get all the candles going again, and it was just really sweet. And I don't have meals like that [in the US], you know, and it was just . . . a central part to our day-to-day that I think was just really special...

\section{Theme 3: Experiencing Threat and Violence}

During the focus group, all three participants reflected on their safety, experiences of violence and threat, and lack of support after these experiences while abroad. One participant, who noted that they are often perceived as a woman by others, explained that they were fearful to travel alone 
during their time abroad because women are susceptible to violence. They expressed regret in this regard, as they described traveling alone as empowering but did not allow themselves to have this experience due to fear of violence.

Along with this fear of potential violence, all participants described experiences of sexual harassment and/or violence while studying abroad. These experiences included sexual harassment and assault, as well as rape. Two participants identified the perpetrators of the harassment and/or violence as LGBTQ+ individuals; one did not disclose the identity of their perpetrator(s). One participant noted that they were more adversely affected by the events following their rape than by the rape itself. These events included being shamed by their partner and various medical and government institutions.

So while I was [at the Embassy to report the rape]... I was just being shamed in that, like, okay, you're a man. Why didn't you fight them off? Or, are you not strong enough? Or, why were you drinking with them? What were you wearing? Or, you do know that gay men are at a higher risk ... of contracting STIs or HIV and whatnot. And I'm just sitting there thinking, like, what the hell does any of this have to do with the fact that I was just raped?

Another participant reflected on the ways in which they were dismissed by their friends after their experience of violence:

... everyone just kind of blew me off, like didn't really ask if I was okay or didn't really hold that for me ... and I do think of it in terms of gender sometimes where I'm like hmm, is that why you're blowing me off, because you don't think it's a big deal because I'm not a ciswoman and it's not a cisman [harassing me]? ... So it just felt very, just like, not good ... And was confusing, and it just kind of felt like I wasn't being taken seriously when trying to talk about it or process it.

The reactions of both friends and institutions to participants' experiences of sexual violence demonstrate the gendered expectations that others held surrounding participants' identities and behaviors, and the verbal policing which occurred as a result. One participant stated that genderpolicing was more violent in their host country than in the United States, although trans and gender expansive people experience daily microaggressions both in the US and abroad.

[My gender and sexuality were] policed, I feel like more violently over there as opposed to [the US] ... Undoubtedly it was mainly men [doing the policing] and it was honestly, mainly queer men. It was a very interesting dynamic that I noticed.

\section{Theme 4: Managing Others' Assumptions About Identity}

Participants discussed the ways they were affected by others' assumptions about their gender and sexual identities during their study abroad experiences. One individual felt forced to 'come out' as sapiosexual/pansexual frequently because others, including their host mom, assumed they were gay:

Because of the way I performed my gender [my host mom] just kind of assumed that I was gay. While I was [abroad] I went on a couple of dates with this one girl at one point and when I told my host mom, she was shocked. She was like, "Wait, what? You went on a date with a girl?" ... So yeah, it was, it was just funny. It was interesting. 
Another participant explained that they felt pressure to 'come out' to strangers while they were abroad due to assumptions about their sexuality:

I noticed, just in my experience too, passing as more feminine, men thinking then that I was kind of straight by default. And that was something that I felt like, kind of the aggression ... with approaching me ... and kind of being in this position where I'm having to, you know, 'out' myself in front of these, these strangers and then, there was different ways that I would say that I wouldn't be interested . . . but I did notice just the overt aggressiveness of it that I feel like I don't get as much of [in the US] ...

Along with gender and sexuality, all participants mentioned that their racial identities affected the ways they were seen and, therefore, treated by others while abroad.

When I kind of reflect [on] . . situations that I'm in or how I'm being read or the dynamics and stuff, I felt like more than my transness a lot of times I was thinking about my whiteness and my like, just being ultimately a tourist from the US. So I feel like, there was just that layer of like, I don't really know how a lot of people are reading me ...

\section{Theme 5: Performing and Policing Gender}

Participants articulated the processes of learning which gender performances were acceptable in the various international locations they visited while abroad and the ways their own gender performances did or did not reflect these cultural expectations. Some participants noted that their gender expressions were dependent on the cultural norms and/or the identities of those by whom they were surrounded in specific instances.

... [Experiencing microaggressions] makes me question a lot about how I'm being perceived, and it even makes me question how I perform, like, the complexities of my identity and how I perform them ... . with different populations. Like, I'm going to perform it differently if I'm around cis men or . . . cis women versus you know, even, like, cis straight men versus queer cis men, you know? So there's a lot of fluctuation there.

One participant recognized the historical context that was responsible for the dynamics of gender 'acceptability' that they observed in their host country:

I could tell that socially transwomen [in my host country] played a very different part than a transmasculine person would play because it just, there's like a whole culture and other history with transwomen there ... So it kind of felt like transmasculine people there sort of had this privilege of more, like, blending in . . . so it felt like just all of it, harassment, policing, everything, like that was way more heavily put on transwomen.

One participant was comforted that their feminine style of dress, which is often stigmatized in the US, blended in in their host country because of cultural differences in regard to gender expectations. Another acknowledged the privilege of 'passing' as cisgender while abroad:

I'll say that I did just feel really privileged in kind of being able to like, that I didn't stand out really in any way and that I could just 'pass' ...

Theme 6: Lacking Preparation and Institutional Support 
Participants described the ways gender and culture intersected with respect to their experiences within international educational settings, as well as the support (or lack thereof) provided to them by educational institutions in the United States and abroad. None of the participants received information or resources from their institutions regarding LGBTQ+ communities or safety considerations for queer students before they traveled abroad. Participants also completed little to no research on their own about their study abroad locations before going. All participants recommended that trans and gender expansive students who plan to study abroad would benefit from researching queer communities in their host countries beforehand.

I didn't really do any kind of research and I remember even trying to find a queer bar in [host city] and I think there was one, maybe ... I wish I would have known more about the communities that do exist in those places. I think that would have enhanced my experience in a lot of ways.

One participant advised that students ask their colleges/universities to provide them with relevant information regarding LGBTQ+ populations in their host country:

I would spend time looking at [LGBTQ+] history in your host country. This might help you understand the way you/your expression are perceived there and perhaps help you feel more comfortable. Neither my home or abroad campus provided me with information regarding the $\left[\mathrm{LGBTQ}^{+}\right]$culture in my host country. If this is the case for most schools, I would advise asking your school for those resources. Communicate its importance (if it's important to you).

Some participants also described positive experiences with specific professors who respected their identities abroad as well as accommodations provided by their institutions:

I lived in a room by myself and that was because I, before going abroad, I went to the Office of Study Abroad [in the US] and explained, like, "I'm trans, so I don't know what you're trying to do with that situation." Because ... from what we were told it seemed more strict [abroad] like girls roomed with girls and boys roomed with boys... and then they offered me a couple different scenarios and one of them was that I could have a single [room] . . and I was like, "Oh, that's tight. I'll do that." ... To just like avoid any sort of discomfort.

\section{Theme 7: Celebrating Their Experiences of Studying Abroad}

Participants reflected fondly on studying abroad and shared specific experiences that deeply moved them. During their follow-up interview, one participant emphasized that an important aspect of their study abroad experience was resilience in the face of adversity. Similarly, although all participants recounted instances of hardship, each stated that they would study abroad again if given the chance.

I'd be more brave about [studying abroad again] ... I think I would push myself, push myself more and I think, yeah. Europe is just, it's very white and it's very 'safe' and comfortable and familiar and, you know, still very privileged to have been given the opportunity to go there, but yeah . . . I think I would go somewhere completely different...than what feels 'safe'.

Each participant shared what they considered the best experience they had while studying abroad. One participant reflected on a camping trip: 
... it was literally on the beach, so it's like the coast and then just mountains and trees on the other side ... we intended to rent a tent but we just kind of kept forgetting and then it was night and everything was closed [laughter] so we ended up sleeping on the sand, under the stars. It was just very magical and just cool and then woke up with the sun ... It was just, very simple in a lot of ways and just very peaceful and magical.

Another participant shared an experience of traveling alone:

... I was just by myself in the forest, just me and the forest. It was really cold, I was barefoot most of the time and almost got frostbite, it was, I'm not sure I really can describe how it felt. It just felt so alive. I felt a very strong connection to the earth, I felt her energy just radiating, just pulsing from, like I could feel her underneath my feet. It was so, she was so alive and it just felt so organic ... It had been a really long time since I had felt that.

\section{Discussion \& Recommendations}

The seven themes yielded from participants' responses emphasize the unique opportunities for increased self-understanding, community-building, and complex reflection regarding gender and culture that characterize the study abroad experiences of trans and gender expansive (TGE) students in this study. In addition, these themes highlight particular burdens of study abroad for TGE populations, including lack of support and connection, gender-policing, violence, and harassment. Bryant and Soria (2015) argue that "those working in higher education can ensure LGBTQQ students have a successful and fulfilling study abroad experience" if provided with the appropriate resources and support (103). If institutions were to advocate for TGE study abroad students in this way, it is possible that this would cause them to feel safer and more confident when going abroad and therefore increase the social accessibility of the study abroad experience for gender minorities. In addition, Bryant and Soria (2015) posit that campuses that provide LGBTQ+ study abroad information and support "may help students to feel more included within their college experience" (101). In this way, colleges that prioritize LGBTQ+ safety and wellbeing in the context of study abroad may increase students' comfort both abroad and at their home campus.

Therefore, in this section, TGE-specific considerations for both prospective study abroad students and colleges and universities are presented. Potential resources and methods of support, which may enhance international experiences and increase social accessibility of study abroad for TGE students, are discussed.

\section{Housing}

In order to provide adequate support to TGE study abroad students, specific aspects of the study abroad experience should be examined. For example, international living arrangements may pose unique challenges to the safety and comfort of TGE students. During the focus group and follow-up interviews, participants shared some of the complex issues they encountered because of their identities while living with host families and among other students. Marijuan and Sanz (2018) suggest that living with host families can provide unique social, cultural, and linguistic opportunities for study abroad students. Bonds created between students and their host families can be intensely gratifying and, in some cases, long-lasting. However, new and unfamiliar living situations may elicit intense fears of misunderstanding, rejection, and even violence for TGE students who are placed with host families. During the host family selection process, universities may want to directly inquire 
about families' level of openness to and understanding of trans and gender expansive communities so that these students may be placed in safe, welcoming home environments. In addition, some study abroad programs provide on- or off-campus student housing rather than host family placement. These living arrangements may also pose safety risks for TGE students, as they are often gender-segregated. In regard to this form of housing, we recommend that institutions provide alternatives (such as single rooms and bathrooms) for students who may not be safe in these shared living spaces.

\section{Community}

Using the transgender resilience intervention model (TRIM), Matsuno and Israel (2018) suggest that specific resilience factors can "buffer the negative influence" of minority stressors which may adversely affect TGE individuals (638). TRIM specifically applies to psychologists and their therapy relationships with trans clients but is discussed more broadly here. One participant in the current study explicitly identified resilience as a method through which they were able to move past experiences of adversity and "even go as far as thriving" during their study abroad experience. Colleges and universities can encourage and create opportunities to promote resilience for TGE study abroad students in order to mitigate the negative mental and physical health consequences caused by minority stressors. Resilience can be enhanced through community, group, and individual interventions (Matsuno and Israel, 2018), although only community and group interventions will be discussed here. Resilience may be promoted in community environments (such as colleges and universities) through the development of gender and sexuality education programs for students, faculty, and administration. The resilience factors within study abroad community environments may also be increased through the creation of LGBTQ+ support groups and student organizations.

Group interventions "are situated to target group-level resilience factors such as social support, family acceptance, community belonging, and finding, as well as being, a positive role model" (Matsuno \& Israel, 2018, p. 643). The authors suggest various ways to help trans individuals create connections with their communities in the context of therapy. Drawing on this idea, study abroad programs may want to provide TGE students with access to community through in-person and online LGBTQ+ study abroad social groups through which students can share experiences and resources, ask questions, practice activism, and form relationships. It is also important that individuals' privacy is protected within these community groups.

All participants in this study reported that they did not receive any LGBTQ+-specific information about their host countries and cultures from their institutions before or during their study abroad trips. Universities could also provide support in the form of LGBTQ+ student advisory committees or student mentors within their institutions to help students understand and prepare for the unique experiences they may have while abroad. Institutions can work closely with LGBTQ+ (and, more specifically, trans) community organizations in order to identify how they may be of service to their trans and gender expansive study abroad students, especially in regard to connecting them with queer communities abroad.

\section{Safety}

In addition to access to community, this study emphasizes the need for increased safety considerations for TGE study abroad students. All three participants shared experiences of sexual 
harassment and/or violence while abroad. This is consistent with findings from the U.S. Transgender Survey, which found that $47 \%$ trans individuals are sexually assaulted at some point in their lifetime (2015). Unfortunately, two participants shared that their experiences of sexual harassment and violence were made worse by the reactions of invalidation and shame they received from friends, partners, medical centers, and government institutions. Byant and Soria (2015) suggest that LGBTQ+ students should receive resources regarding safety considerations specific to their host country. Similarly, colleges and universities could take extra precautions to ensure that TGE students are protected from sexual violence and that they have adequate resources and support to help them report and heal from these events during and after study abroad.

\section{Reflection}

Although all three participants in the current study endured extreme hardship during their time abroad, when asked if they would study abroad again, each answered affirmatively and without hesitation. During the focus group, participants reflected on their many positive international experiences, including forming strong interpersonal bonds, visiting incredible new places, and gaining important insights about themselves and the world. The concepts of resilience, fun, and connection were all crucial aspects of the study abroad experiences participants shared during the focus group and follow-up interviews. A 2011 study examining trans experiences found that $90 \%$ of trans participants reported at least one positive aspect of having a trans identity (Riggle et al., 2011). The positive experiences stemming from having a trans identity included congruency between identity and expression/appearance, personal growth and resiliency, empathy, interpersonal relationships, unique perspective/insight, living beyond the binary, activism, and community (Riggle et al., 2011).

At some point after the focus group or during the follow-up interviews, each participant expressed to the facilitator that they appreciated the opportunity to be involved in the study, and that they found the research to be important. One participant shared: "Yeah, and [participating in this research is] helpful for me because I feel like this is something that I haven't really openly talked about with anyone about and also, it feels foreign to me to be having these conversations. Like, I found myself feeling intimidated at moments during the discussion with the group and not in a negative way at all but just that, it's interesting that this is something that I'm just kind of beginning to explore, I feel like. Like, I really do feel, yeah, like it's just kind of the beginning of this conversation... So, I appreciate you." Participants shared that they were not provided with any sort of university-sponsored opportunity for reflection with other study abroad students after their return to the US.

As participants appreciated this study, in part, because it allowed them to process and reflect on their international experiences in the context of their identities, institutions might consider providing students with the opportunity to reflect on the joys, complexities, frustrations, and sorrows of their travels abroad. This can be a chance to consolidate learning through discussions with others. TGE students can safely process and celebrate their unique experiences abroad with other students who may have had shared experiences.

\section{Student Preparation}

Each participant shared that they did little to no research about LGBTQ+ communities, history, and safety on their own before traveling abroad, and two expressed that they wish they would have 
been more prepared in this way. While institutions are obligated to provide all students with resources that will help them have safe, enriching international experiences, students should be encouraged to engage in their own research about their host country before studying abroad. Institutions can help make this information more accessible and can encourage students, including LGBTQ+ students, to be self-directed in preparing for studying abroad. As students often understand their own needs more fully than their institutions do, it is imperative that they also actively prepare to live in an unfamiliar culture by increasing their own understandings of the ways their identities may specifically impact their cultural experiences.

The current study has identified that trans and gender expansive students may experience unique challenges and joys while studying abroad as a result of the complex interactions between gender, culture, language, and identity. Institutions are responsible for the safety and wellbeing of their study abroad students, and should provide specific resources and support to help trans and gender expansive students thrive.

\section{Limitations}

This pilot study was limited by its small sample size of three participants and specific location of one U.S., Midwestern city. Due to the sample size, researchers were only able to conduct one focus group, although more are required to provide an encompassing narrative of trans and gender expansive students' study abroad experiences. This study is intended to be explorative in nature and seeks to identify issues for further research. In addition, as two participants studied in Europe and one in Asia, the current project is not designed to draw specific conclusions about the intersections of gender and culture in a particular place. Rather, it is the hope of the researchers that this pilot study provides a window into study abroad experiences as a whole for trans and gender expansive students. Further research may identify the nuanced relationships between TGE identity and particular cultures around the world. Finally, the first author has a personal relationship with two of the three participants (although the other researchers have no personal relationships with participants), which may have affected participants' participation and willingness to share specific details of their experiences.

\section{Conclusion}

The current study identified seven themes within the experiences of three trans and/or gender expansive undergraduate students who have studied internationally. These themes provide insight into the unique challenges these TGE students faced, as well as the rewards they experienced studying abroad. It is the hope of the authors that these findings will be utilized by undergraduate institutions in the creation and provision of support and resources specific to gender minority students. As existing research on trans and gender expansive study abroad experiences is scarce, this study provides a shared narrative for TGE students on this complex topic and identifies areas for future research and exploration. Study abroad is a time for personal, professional, and educational growth that many students remember for the rest of their lives. It is crucial that this experience is accessible to trans and gender expansive students so that they may be afforded this potentially lifealtering opportunity free from isolation, violence, and threat. 


\section{References}

Braun, V., \& Clarke, V. (2006). Using thematic analysis in psychology. Qualitative Research in Psychology, 3(2), 77-101. doi:10.1191/1478088706qp063oa

Bryant, K. M., \& Soria, K. M. (2015). College students' sexual orientation, gender identity, and participation in study abroad. Frontiers: The Interdisciplinary Journal of Study Abroad, 25, 91-106.

Cisneros-Donahue, T., Krentler, K. A., Reinig, B., \& Sabol, K. (2012). Assessing the academic benefit of study abroad. Journal of Education and Learning, 1(2), 169-178. doi:10.5539/jel.v1n2p169

Clarke, V., \& Braun, V. (2013) Teaching thematic analysis: Overcoming challenges and developing strategies for effective learning. The Psychologist, 26(2), 120-123. ISSN 0952-8229

Crocker, J., \& Major, B. (1989). Social stigma and self-esteem: The self-protective properties of stigma. Psychological Review, 96(4), 608-30. doi:10.1037/0033-295x.96.4.608

Farmer, M. (2017). Study abroad participation by state and demographics data. Washington, DC: NAFSA: Association for International Educators. Retrieved from www.nafsa.org

Farrugia, C., \& Sanger, J. (2017). Gaining an employment edge: The impact of study abroad on 21st century skills \& career prospects in the United States. New York, NY: Institute for International Education Center for Academic Mobility Research and Impact. Retrieved from www.iie.org

Hendricks, M. L., \& Testa, R. J. (2012). A Conceptual framework for clinical work with transgender and gender nonconforming clients: An adaptation of the minority stress model. Professional Psychology: Research \& Practice, 43(5), 460-467. doi:10.1037/a0029597

James, S. E., Herman, J. L., Rankin, S., Keisling, M., Mottet, L., \& Anafi, M. (2016). The report of the 2015 U.S. Transgender Survey. Washington, DC: National Center for Transgender Equality. Retrieved from www.ustranssurvey.org

Marijuan, S., \& Sanz, C. (2018). Expanding Boundaries: Current and new directions in study abroad research and practice. Foreign Language Annals, 51(1), 185-204. doi:10.1111/flan.12323

Matsuno, E., \& Israel, T. (2018). Psychological interventions promoting resilience among transgender individuals: Transgender resilience intervention model (TRIM). The Counseling Psychologist, 46(5), 632-655. doi:10.1177/0011000018787261

Meezan, W., \& Martin, J. (Eds.). (2009). Handbook of research with lesbian, gay, bisexual and transgender populations. New York, NY: Taylor \& Francis.

Meyer, I. H. (1995). Minority stress and mental health in gay men. Journal of Health and Social Behavior, 36, 38-56. doi:10.2307/2137286

Meyer, I. H. (2003). Prejudice, social stress, and mental health in lesbian, gay, and bisexual populations: Conceptual issues and research evidence. Psychological Bulletin, 129, 674-697. doi:10.1037/00332909.129.5.674

Meyer, I. H. (2015). Resilience in the study of minority stress and health of sexual and gender minorities. Psychology of Sexual Orientation and Gender Diversity, 2(3), 209-213. doi:10.1037/sgd0000132

Riggle, E. B., Rostosky, S. S., McCants, L. E., \& Pascale-Hague, D. (2011). The positive aspects of a transgender self-identification. Psychology \& Sexuality, 2(2), 147-158. doi:10.1080/19419899.2010.534490

Testa, R. J., Habarth, J., Peta, J., Balsam, K., \& Bockting, W. (2015). Development of the Gender Minority Stress and Resilience Measure. Psychology of Sexual Orientation and Gender Diversity, 2(1), 65-77. doi:10.1037/sgd0000081 


\section{Appendix: Focus Group Question}

1. Based on your personal experience, how do you think transgender and gender expansive people are treated in the United States? How does this compare to how this population is treated in your host country?

2. How did you prepare for your trip and how did you feel before leaving to study abroad?

3. In general, how, if at all, do you feel that your gender affected your experience during your study abroad trip?

4. How would you describe the relationship(s) you had abroad, especially in the context of your gender? This can include your housemate(s), friends, romantic/sexual partners, etc.

5. What was your experience with educational settings abroad, and were you 'out' at school? This can include discussion about teachers, classes, classmates, schoolwork, etc.

6. In general, how would you compare the decision to 'come out' and/or the process of 'coming out' in the U.S. versus in your host country?

7. Did you have relationships (of any kind) with transgender and/or gender expansive people in your host country? How did this change/would this have changed your experience, if at all?

8. How, if at all, did your gender affect your decisions about whether or not to travel while you were abroad, and your decisions about your travel destinations?

9. What was the worst experience you had while studying abroad?

10. What was the best experience you had while studying abroad?

11. Have the ways in which you think about your own or other people's genders changed since you returned from (or as a result of) studying abroad? (omitted from interview due to time constraint)

12. If you had the opportunity, would you study abroad again? Why?

13. What advice would you give to other trans and gender expansive students who are considering studying abroad? 